\title{
Shear-induced organization of forces in dense suspensions: signatures of discontinuous shear thickening
}

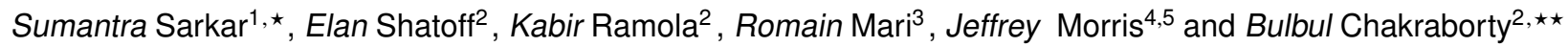 \\ ${ }^{1}$ Physics of Living Systems, MIT, 400 Technology Square, Cambridge, MA 02139, USA \\ ${ }^{2}$ Martin Fisher School of Physics, Brandeis University, Waltham, MA 02454, USA \\ ${ }^{3}$ Department of Applied Mathematics and Theoretical Physics, Centre for Mathematical Sciences, University of Cambridge, Cam- \\ bridge CB3 OWA, UK \\ ${ }^{4}$ Benjamin Levich Institute, City College of New York, New York, NY 10031, USA \\ ${ }^{5}$ Department of Chemical Engineering, City College of New York, New York, NY 10031, USA
}

\begin{abstract}
Dense suspensions can exhibit an abrupt change in their viscosity in response to increasing shear rate. The origin of this discontinuous shear thickening (DST) has been ascribed to the transformation of lubricated contacts to frictional, particle-on-particle contacts. Recent research on the flowing and jamming behavior of dense suspensions has explored the intersection of ideas from granular physics and Stokesian fluid dynamics to better understand this transition from lubricated to frictional rheology. DST is reminiscent of classical phase transitions, and a key question is how interactions between the microscopic constituents give rise to a macroscopic transition. In this paper, we extend a formalism that has proven to be successful in understanding shear jamming of dry grains to dense suspensions. Quantitative analysis of the collective evolution of the contactforce network accompanying the DST transition demonstrates clear changes in the distribution of microscopic variables, and leads to the identification of an "order parameter" characterizing DST.
\end{abstract}

\section{Introduction}

A remarkable property of dense stabilized suspensions of particles in the tens of nanometers to tens of micrometer size range is that they can abruptly transform from a low to a high viscosity phase (or even a solid-like phase) with increasing applied stress [1-5]. Under steady shear, these suspensions undergo a discontinuous shear thickening (DST) transition [1-3]. A confluence of ideas from the granular and fluid dynamics communities has led to new understanding and a new set of questions regarding the flowing and jamming behavior of dense suspensions $[3,6,7]$. In this paper, we analyze the DST transition using a force-space representation [8] that was originally developed for granular systems. We extend the formalism to suspensions and identify distinct, quantitative signatures of the DST transition in this representation.

There is developing consensus that particle contact and friction play a crucial role in dense suspension rheology $[9,10]$. Combining fluid mechanical interactions with contact friction between particles has been shown to capture critical features of both DST [11-14] and shear induced jamming (SJ) [15]. Furthermore, clear connections have been made between a dynamic shear jamming front and impact-driven solidification [5]. The appearance of non-monotonic flow curves in numerical models

\footnotetext{
$\star$ e-mail: sumantra@mit.edu
}

$\star \star$ e-mail: bulbul@brandeis.edu of DST [11, 12] is reminiscent of classical phase transitions. As in classical phase transitions, the key question is how interactions between the microscopic constituents give rise to a macroscopic transition.

The essential idea underlying the jamming-based rheology model $[3,6]$ for DST is that it requires a shearstress driven transition from the lubricated to the frictional branch of the viscosity. Reduction of the jamming packing fraction through the creation of frictional contacts [7] yields a higher viscosity at any $\phi$. If shearing changes the relative fractions of frictional and lubricated contacts, the suspension can transition to the frictional branch. We are interested in understanding the nature of the collective reorganization of particles that gives rise to this change in rheology. Simulations show that DST is accompanied by significant changes in the network of frictional contacts and contact forces with minimal changes in structure factors and pair correlation functions [2]. We present a quantitative analysis of changes in the force network by studying the organization in "force space", which is dual to the positional network of grains in a sense to be defined below $[8,16]$. The density of points in this space naturally decreases as the stress increases since the number of contacts is roughly constant, but more importantly we show that the rheological behavior characterizing DST is accompanied by changes in the form of the density distribution of points in this space. The change in distribution is a consequence of the constraints of local mechanical equi- 
librium applied to grains as contacts change from lubricated to frictional.

\section{Model}

The combination of frictional and viscous interactions between surfaces of suspended particles has recently been addressed through simulations [11-14] that use a hybridization [11, 12] of Stokesian Dynamics (SD) with discrete-element modeling (DEM) [17]. The SD method is simplified by only considering the near-field, or lubrication, hydrodynamic interactions. To allow contact, the lubrication resistance singularity is cut off at a distance $h_{c}$ between particle surfaces. The DEM approach uses a Coulomb friction law: for $F_{\tan } \leq \mu F_{n o r m}$, where $\mu$ is the interparticle friction coefficient, there is no slipping of the contact, while there is slip when the friction is fully mobilized for $F_{\tan }=\mu F_{n o r m}$. To model the transition from lubricated to frictional contacts, a repulsive force, representative of electrostatic repulsion or steric hindrance due to a grafted layer, is used. For low stress or low shear rate, the repulsive force maintains surfaces separated at $h>h_{c}$ and thus interactions are lubricated, as if $\mu=0$, while at large enough stress to push particles to $h<h_{c}$, frictional contacts are activated. 2D simulations of this model capture the progressively steeper shear thickening with increasing $\phi$ (Fig. 2a) seen in experiments.

\section{Force Balance in 2D Suspensions}

Owing to the size $(\leq 10 \mu \mathrm{m})$ of the particles and the shear rates at which DST is observed (usually around $1 s^{-1}$ ), it is a very low Stokes number phenomenon, and particle inertia can be neglected. As a consequence, the constraints of mechanical equilibrium are strictly obeyed at both the local and global levels. We analyze data obtained from stress-controlled simulations of a bidisperse suspension of particles using the hybrid SD-DEM model $[11,12]$ in 2D. Each disk in the suspension experiences a hydrodynamic force, $\vec{F}_{H}$, a short range repulsive force, $\vec{F}_{R}$, and frictional contact force, $\vec{F}_{C}$. The hydrodynamic component includes a non pairwise force (Stokes drag) and pairwise forces (lubrication), whereas $\vec{F}_{R}$ and $\vec{F}_{C}$ are pairwise interactions. The force balance equation for each disk is $\vec{F}_{H}+\vec{F}_{R}+\vec{F}_{C}=0$. The repulsive force is modeled as a short range electrostatic repulsion,

$$
\vec{F}_{R}^{(i, j)} \propto \begin{cases}-\frac{a_{i} a_{j}}{a_{i}+a_{j}} e^{-\kappa h^{(i, j)}} & h^{(i, j)} \geq 0 \\ -\frac{a_{i} a_{j}}{a_{i}+a_{j}} & h^{(i, j)} \leq 0,\end{cases}
$$

with Debye length, $1 / \kappa$, much smaller than the radius of either particle, $a=\left(a_{i}\right.$ or $\left.a_{j}\right)$. Adjacent grains that overcome this repulsive force form frictional contacts and contribute to the $\vec{F}_{C}$. Thus, $\left|F_{R}\right|$ sets a stress scale, $\sigma_{0} \approx \frac{\left|F_{R}\right|}{a^{2}}$, whose interplay with the hydrodynamic stress scale determines the stress (shear rate) dependent rheology.
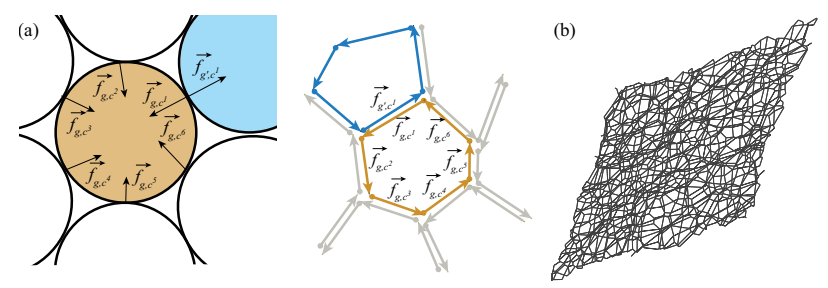

Figure 1. (a) Construction of force tiles: The vectors $\vec{f}_{g, c}$ form a closed polygon (light brown) (Eq. 2), and there is strict edge sharing between grains $g$ and $g^{\prime}$ (Eq. 3). (b) A force tiling constructed from a configuration at $\sigma=\sigma_{x y} / \sigma_{0}=0.1$. The shape and size of the bounding parallelogram is determined by the components of the imposed stress tensor: $\sigma$ controls the opening angle. The number of tiles is equal to the number of particles in the force-bearing network

\subsection{Force tilings in granular materials}

In packings of dry grains, labeling the contacts of grain $g$ by $(g, c)$ (Fig. 1), the force balance condition is:

$$
\vec{F}_{C} \equiv \sum_{c} \vec{f}_{g, c}=0
$$

where the sum is taken over all the contacts $\{c\}$ for a given grain $g$. In addition, Newton's third law states:

$$
\vec{f}_{g, c}=-\vec{f}_{g^{\prime}, c} .
$$

These constraints can be used to construct a representation known as the "force tile" representation or MaxwellCremona tiles [18]. Eq. (2) can be represented as a closed polygon, if the sum is taken cyclically over the contacts for each grain. Eq. 3 dictates that the tiles of touching grains have to share an edge. We can, therefore, construct a force tiling, as illustrated in Fig. 1. The vertices of the force tiling form a point pattern. In previous studies of the shear-jamming process in dry granular systems, it has been shown that the onset of shear-jamming is marked by distinct changes in this point pattern $[8,16]$.

\subsection{Generalization to Suspensions}

The Stokes drag is a non-pairwise force that prevents individual force tiles from closing: $\sum_{c} \vec{f}_{g, c}=\vec{f}_{\text {Stokes }}$. In addition, suspended grains experience pairwise, non-contact forces. The latter can be included by extending the definition of the contact network, $\{g, c\}$, and $\left\{\overrightarrow{f_{g, c}}\right\}$ to include $\vec{F}_{R}$, $\vec{F}_{c}$ and the pairwise lubrication force. Given these, and $\vec{f}_{\text {Stokes }}$, there is a unique way of obtaining effective contact forces such that all force tiles close [19]. We use an iterative algorithm [8] in which the contact forces are modified to ensure that Eqs. 2 and 3 are satisfied at every iteration. The final solution is unique up to global translation.

\section{Results}

We construct force tilings for each member of the ensemble of steady states over a range of shear stresses, 
$\sigma \equiv \sigma_{x y} / \sigma_{0}$ and at packing fractions, $\phi=0.76$ and 0.78 . The point patterns of force-tiling vertices from three representative configuration at $\sigma$ below and above the DST transition for $\phi=0.78$ are shown in Fig. 2. The lengths of the boundaries increase with increasing $\sigma$, and they have been scaled by $\sigma$ in order to aid in the visualization of the patterns. It should be pointed out that the shape of the bounding boxes fluctuate even at a given $\sigma$, which fixes only the opening angle. Fig. 2 (b) illustrates the effect of increasing $\sigma$ on the density of vertices. This density is observed to follow $\rho_{v} \propto 1 / \sigma^{2}$ for up to six decades, and occurs as $\phi$ is held constant with no change in the realspace density. Given the dramatic change in $\rho_{v}$ across the DST, one could expect significant changes in the patterns of vertices. We have employed a clustering analysis of these point patterns based on purely local densities. In the next section we demonstrate that there is a clear signature of the DST transition in the clustering properties of the vertices.

\subsection{Point Patterns of Vertices}

(a)

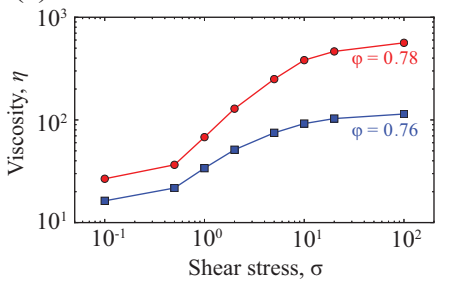

(b)

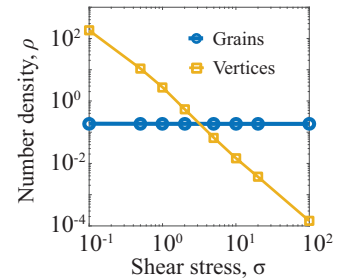

Figure 2. (a) Viscosity as a function of shear stress in stresscontrolled simulations of a non-Brownian suspension. (b) Density of grains and vertices as a function of shear stress.

\subsubsection{Density based clustering analysis}

If the repulsive force between two contacts is overcome to create a frictional contact, the constraints of mechanical equilibrium will necessarily induce a change in the shape of the force tiles, and hence a collective reorganization of the point pattern of vertices. We performed a density based clustering analysis of the vertices of force tiles using the DBSCAN algorithm [20] to detect these changes. In DBSCAN, two points belong to the same cluster if their distance is less than a probing length scale, $l$ (which here will dimensionally be a stress scale). The connected set of all such points define a unique cluster, which are separated from each other by distances greater than $l$. In usual implementations of DBSCAN, clusters are determined using an optimum probing radius.

We use DBSCAN to analyze how the clustering pattern changes as the probing length scale is varied. For a large enough length scale, all points will belong to one cluster and for small enough length scales, each point will be its own cluster. Our algorithm probes the density distribution of point patterns at different length scales by monitoring the number of clusters, $N_{c}(l)$, as a function of the probe length, $l$. For a point pattern with uniform density, $N_{c}(l)$ decreases continuously with $l$. In a periodic lattice, where the distance distribution of nearest neighbors is a delta function, $N_{c}(l)$ exhibits a jump discontinuity at the lattice spacing. For a complex pattern, we expect $N_{c}(l)$ to show significant changes in its derivatives at length scales where the distance distribution has structure.

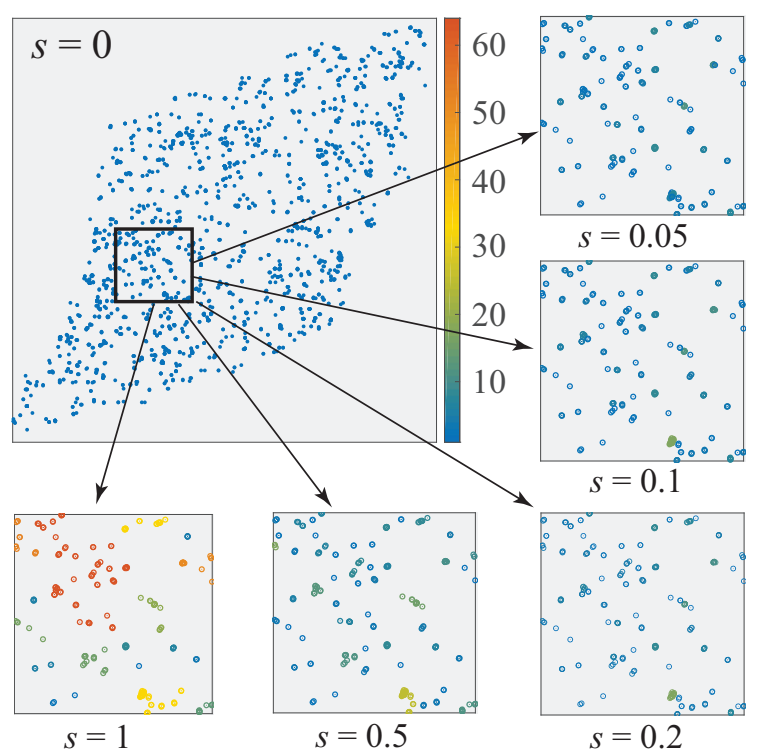

Figure 3. Illustration of DBSCAN analysis. Left: Sample tiling at $\phi=0.78, \sigma=100$ at $s=0$ : all clusters are of size 1 and $N_{c}(s=0)=N_{v}$, the number of vertices. Right panel: Zoomed in point pattern analyzed with increasing probe length $s$. The clusters are colored by their size as indicated by the color bar.

In applying the clustering analysis to point patterns in force tilings, the probing "length scale" is to be interpreted as an isotropic probing stress scale, $\sigma_{\text {probe }}$. We measure the variation in the number of clusters $N_{c}(s)$ as the dimensionless probe stress, $s=\sigma_{\text {probe }} / \sigma$, is increased (Fig. 3). Identifying significant variations in $N_{c}(s)$ yields characteristic stress scales in the patterns, just as in real-space.

\subsubsection{Clustering Analysis of DST}

We calculate $N_{c}(s)$ for each force tiling and then ensemble average over tilings sampled at given $\phi$ and $\sigma$. Fig. 4 (a) shows that at $\phi=0.78$, and $\sigma$ below the DST transition, $N_{c}(s)$ decays continuously with $s$, indicating the lack of any characteristic scale in the distribution of distances between the vertices. We note that vertices that are close to each other are not necessarily connected by an edge in the tiling. Therefore, the distance distribution is not equivalent to the contact force distribution.

Above the DST transition, we can clearly identify three different decay regimes in $N_{c}(s)$, with a plateau-like structure clearly identifiable in the derivative, $d N_{c}(s) / d s$ shown in Fig. 4 (b). As seen from Fig. 4 (c), this structure in $d N_{c}(s) / d s$ is much more pronounced for $\phi=0.78$ than it is for $\phi=0.76$. The observed structure in $N_{c}(s)$ indicates that there are density inhomogeneities in the pattern of the vertices characterized by both $s^{*}$, marking the 
onset of the plateau, and $s^{* *}$, the edge of the plateau. The abrupt change in $d N_{c}(s) / d s$ to a much smaller value at $s^{*}$ implies that incremental changes in $s$ beyond $s^{*}$ incorporate the "noise points": isolated vertices that lie in lowdensity regions. Beyond $s^{* *}, d N_{c}(s) / d s$ starts decreasing as $s$ increases indicating that clusters separated by $s \geq s^{* *}$ are beginning to merge. Fig. 3 illustrates how the cluster pattern changes with $s$. The difference $\Delta s=s^{* *}-s^{*}$ provides a measure of the separation of high density regions in force tilings: the more pronounced the plateau the sharper is the distinction between high and low density regions.

Fig. $4(\mathrm{~d})$ demonstrates that $\Delta s$ increases sharply at a characteristic value of $\sigma$ that depends on $\phi$. Moreover the saturation value of $\Delta s$ decreases with decreasing $\phi$, suggesting that $\Delta s$ can be considered as an "order parameter" characterizing DST.
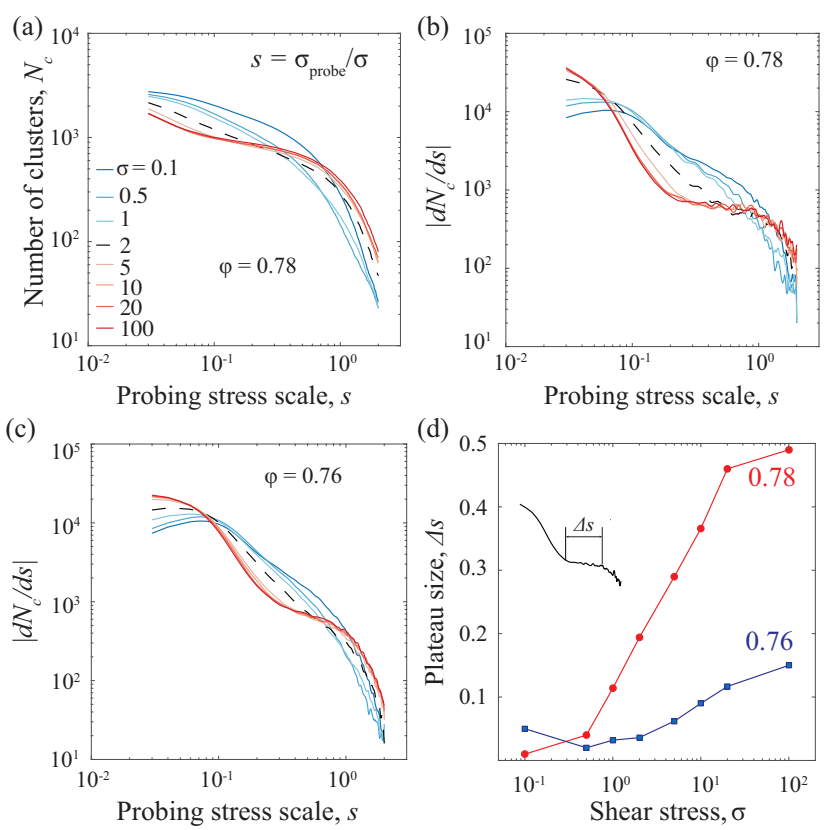

Figure 4. Top: Cluster analysis at $\phi=0.78$ : Number of clusters, $N_{c}$, versus $s=\sigma_{\text {probe }} / \sigma$, at different values of $\sigma$. b) $d N_{c} / d s$ vs $s$. Each curve is obtained by averaging over fifty steady state configurations. The black dashed lines highlight the behavior change at $\sigma \approx 2$. Bottom: (c) $d N_{c} / d s$ vs $s$ for $\sigma=0.1$ and 100 at $\phi=0.76$ compared to $\phi=0.78$. (d) Plateau size $\Delta s$ (See inset for the definition) as a function of the control shear stress $\sigma$ for packing fractions 0.76 and 0.78 .

\section{Discussion}

Using a clustering analysis, we have demonstrated that the rheological changes at DST are accompanied by a collective reorganization in the space of forces. This collective response is necessitated by the constraints of mechanical equilibrium, applied at the local grain level, as lubrication forces change to frictional contact forces. We identify a characteristic stress scale in the pattern, $\Delta s$, from the plateau in $d N_{c}(s) / d s$. This plateau suggests that the vertices are clustered into clumps with an approximately uniform density of points with the clumps separated by $\approx \Delta s$. Equilibrium clumped phases are observed in systems with ultrasoft interaction potentials [21, 22]. Indeed, our preliminary DBSCAN analysis of the clumped phase shows a plateau structure remarkably similar to Fig. 4 (b). We are currently exploring this connection further.

\section{Acknowledgements}

The authors acknowledge useful conversations with Jetin Thomas and Abhinendra Singh. SS, KR and BC acknowledge the support of NSF-DMR 1409093 and the W. M. Keck Foundation. JM and BC acknowledge NSF-CBET 1605283.

\section{References}

[1] E. Brown, H.M. Jaeger, Rep Prog Phys 77, 046602 (2014)

[2] R. Mari, et. al., Journal of Rheology 58, 1693 (2014)

[3] M. Wyart, M. Cates, Phys Rev Lett 111, 218301 (2013)

[4] S.R. Waitukaitis, H.M. Jaeger, Nature 487, 205 (2012)

[5] E. Han, I.R. Peters, H.M. Jaeger, Nat Commun 7, 12243 (2016)

[6] M.E. Cates, M. Wyart, Rheologica Acta pp. 1-10 (2014)

[7] E. Guazzelli, J.F. Morris, A Physical Introduction to Suspension Dynamics (Cambridge University Press, 2011)

[8] S. Sarkar, et. al., Phys Rev E 93, 042901 (2016)

[9] E. Brown, H.M. Jaeger, J. Rheol. 56, 875 (2012)

[10] D. Lootens, et. al., Phys. Rev. Lett. 95, 268302 (2005)

[11] R. Seto, et. al., Phys. Rev. Lett. 111, 218301 (2013)

[12] R. Mari, et. al., J. Rheol. 58, 1693 (2014)

[13] C. Heussinger, Phys. Rev. E 88, 050201(R) (2013)

[14] N. Fernandez, et. al., Phys. Rev. Lett. 111, 108301 (2013)

[15] R. Mari, et. al., Phys. Rev. E 91, 052302 (2015)

[16] S. Sarkar, et. al., Phys. Rev. Lett. 111, 068301 (2013)

[17] P.A. Cundall, O.D.L. Strack, Geotechnique 29, 47 (1979)

[18] B.P. Tighe, T.J.H. Vlugt, Journal of Statistical Mechanics: Theory and Experiment 2011, P04002 (2011)

[19] K. Ramola, B. Chakraborty, submitted to Journal of Statistical Physics

[20] M. Ester,et. al., , AAAI Press, 226, (1996)

[21] J.B. Delfau, et. al., Phys. Rev. E 94, 042120 (2016)

[22] W. Klein, et. al., Physica A 205, 738 (1994) 